\title{
TRÍCH XUẤT ĐƯờNG BỜ BIỂN TÙ ẢNH SENTINEL-1A KHU VỰC THÀNH PHỐ PHAN THIẾT
}

\author{
Huỳnh Yến Nhi ${ }^{1}$ Lê Thị Kim Thoa²
}

Tóm tắt: Trong nhũng năm gần đây, quá trình xói lở, bồi tụ tại khu vực ven biển ngày càng diễn biến phức tạp và có chiều hướng ngày càng gia tăng về tốc độ và phạm vi. Phưong pháp viễn thám được xem là một công nghệ thu thập, xủ̉ lý dũ liệu hũu ich giúp cho các nhà khoa hoc có thể theo dõi hình thái diễn biến đường bò̀ một cách nhanh chóng và liên tục qua thời gian. Trong nghiên cúu này, dũ liệu ảnh rađa Sentinel-1A được sủ dụng nhằm trích xuất thông tin đường bò̀ tại khu vục thành phố Phan Thiết. Ranh giới giũua đất và nước được xác định thông qua quy trình gồm hai bước: phân cưm mò̀ và thiết lập nguoõng. Kết quả rút trích đường bờ tại khu vực nghiên cưu được chuyển sang dạng véc tơ và sau đó so sánh với đường bờ được số hóa thủ công. 350 vị trí được chọn cách đều nhau 100 m để tạo mặt cắt ngang giữa 2 kết quả đường bò̀ nhằm tính toán sự sai khác. Kết quả cho thấy, có 298 vị trí (85\%) có khoảng cách 2 đường bò̀ tù 0 đến $10 \mathrm{~m}$ (1 pixel) và 52 vị trí (15\%) là trên $10 \mathrm{~m}$. Kết quả trên cho thấy khả năng ứng dụng phưong pháp phân cưm mò̀ và thiết lập ngương nhằm rút trích đường bò̀ tụ động trên ảnh vệ tinh Sentinel-1A phục vụ đánh giá nhanh tình hình xói lở, bồi tụ khu vực ven biển hưu hiệu.

Tù khóa: Xói lở/Bồi tụ, Trích xuất đường bò̀, Anh Sentinel-1A, Phân cưm mờ và thiết lập ngương.

Ban Biên tập nhận bài: 12/10/2018; Ngày phản biện xong: 26/12/2018; Ngày đăng bài: 25/01/2019

\section{1. Đặt vấn đề}

Thành phố (TP) Phan Thiết có tiềm năng trong phát triển kinh tế biển với các lĩnh vực như du lịch, cảng biển và nuôi trồng thủy hải sản... Tuy nhiên, nơi đây đang diễn ra tình trạng xói lở và bồi tụ nghiêm trọng do chịu ảnh hưởng của triều cường và sóng lớn. Một số nghiên cứu cũng đã chỉ ra rằng việc xây dựng đê chắn sóng ảnh hưởng đến quy luật xói lở và bồi tụ ở khu vực này $[1,2,3]$. Vì vậy, theo dõi biến động đường bờ là việc làm cần thiết phục vụ cho công tác quản lý và qui hoạch kinh tế xã hội. Đường bờ biển là một trong những đối tượng nghiên cứu năng động nhất của vùng ven biển. Theo Dolan nó được định nghĩa một cách đơn giản theo tính chất vật lý là nơi giao nhau giữa đất và nước [4]. Dưới tác động của các yếu tố hải dương (thủy triều, sóng, gió), cấu trúc địa mạo và các hoạt động kinh tế xã hội của con người, đường bờ thay đổi liên tục theo thời gian [5].

Đường bờ có thể xác định bằng nhiều phương

${ }^{1,2}$ Trường ĐH Tài nguyên Môi trường TP. HCM Email:nhihy@hcmunre.edu.vn pháp khác nhau $[6,7,8]$,trong đó phương pháp viễn thám đã được sử dụng phổ biến hiện nay, bởi tính ưu việt của dữ liệu liên tục vàphạm vi rộng. Năng lượng sóng rađa có khả năng xuyên qua mây, mưa và thu nhận tín hiệu vào ban đêm, phù hợp cho việc giám sát đường bờ, đặc biệt là ở khu vực thường chịu ảnh hưởng bởi mây, mưa. Ảnh rađa thu nhận độ tương phản dựa trên tính chất điện môi và độ nhám bề mặt của đối tượng, từ đó có thể xác định được ranh giới giữa đất và nước $[9,10]$. Trong những năm gần đây, có nhiều công trình tập trung nghiên cứu các phương pháp trích xuất đường bờ từ dữ liệu rađa, với nhiều thuật toán được sử dụng như: phát hiện cạnh (Edge Detection) [11,12,13]; thiết lập ngưỡng (Setting Thresholds) [14,15,16]; mô hình đường viền động (Active Contour Model) [17]... hoặc các phương pháp kết hợp [18,19,20]. Trong đó, hướng tiếp cận logic mờ của Demir và các cộng sự $[21,22]$ đã cho kết quả trích xuất đường bờ với độ chính xác cao. Từ sự thành công này, chúng tôi hy vọng rằng phương pháp phân cụm 
mờ có thể cho kết quả khả quan tại khu vực biển TP. Phan Thiết, Việt Nam.

\section{Khu vực nghiên cứu và dũ liệu}

a) Khu vục nghiên cúu

Khu vực nghiên cứu là vùng ven biển biển của TP Phan Thiết, tỉnh Bình Thuận,với chiều dài khoảng $35 \mathrm{~km}$ (Hình 1 ). Khu vực này nằm trong vùng nhiệt đới gió mùa với hai hướng gió chính là: Gió mùa Đông Bắc (từ tháng 11 đến tháng 4) và gió mùa Tây Nam (từ tháng 5 đến tháng 10 ). Mực nước thủy triều trung bình từ 2 - 3m [23].

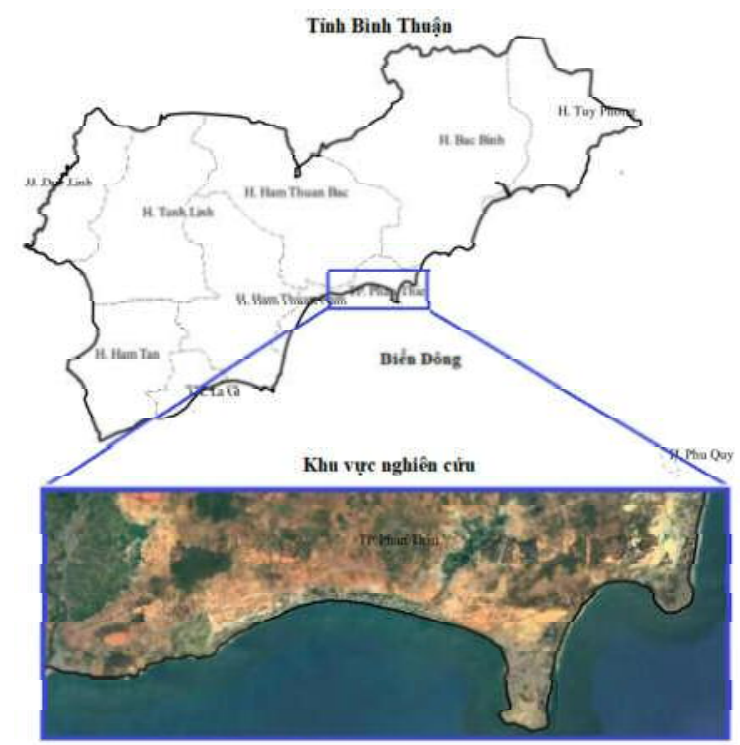

Hình 1. So đồ khu vưc nghiên cứu

\section{b) Dũ liệu}

Ảnh vệ tinh ra đa Sentinel-1A được sử dụng để trích xuất thông tin đường bờ tại khu vực nghiên cứu. Ảnh Sentinel-1A được tải miễn phí từ Cơ quan hàng không vũ trụ châu Âu (ESA). Thông tin của dữ liệu được mô tả tại Bảng 1 .

Bảng 1. Đặc trung của ảnh vệ tinh Sentinel-

\begin{tabular}{ll}
\hline Ngày thu thập & $15 / 11 / 2017$ \\
\hline Thiết bị: & SAR-C \\
Chế độ chụp & IW Swath mode \\
Phân cực & VH, VV \\
Độ phân giải không gian & $10 \mathrm{~m}$ \\
Mức xử lý & 1 \\
\hline
\end{tabular}

Ngoài ra, dữ liệu ảnh vệ tinh độ phân giải cao Google Earth được trích xuất đường bờ bằng phương pháp số hóa thủ công nhằm đánh giá lại kết quả trích xuất đường bờ từ ảnh Sentinel-1A. Dựa vào bảng độ cao mực nước từng giờ tại trạm Vũng Tàu, cho thấy mực nước triều tại khu vực nghiên cứu không biến động nhiều, biên độ giao động giữa nước ròng và nước lớn từ 1-2 $\mathrm{m}$. Đồng thời, tại khu vực ven biển TP. Phan Thiết chiều rộng bãi triều tương đối hẹp (trung bình 15 - 20 $\mathrm{m}$ khi triều thấp) [2], một số nơi có độ dốc lớn và có kè bảo vệ. Vì vậy, ảnh vệ tinh Google Earth (10/10/2017) phù hợp để đánh giá độ chính xác kết quả trích xuất đường bờ từ ảnh Sentinel-1A có độ phân giải không gian 10m.

\section{Phương pháp nghiên cứu}

Trong bài viết này, chúng tôi sử dụng phương pháp phân cụm mờ để trích xuất thông tin đường bờ khu vực TP. Phan Thiết. Đặc điểm của phương pháp này là dựa trên cường độ và tính chất cấu trúc của đối tượng được thu nhận từ kỹ thuật InSAR (Rađa khẩu độ tổng hợp giao thoa). Sau đó, kết quả trích xuất đường bờ tự động từ phương pháp phân cụm được so sách với đường bờ được số hóa bằng phương pháp thủ công.

Quy trình xử lý dữ liệu Sentinel-1A IW GRDH bao gồm các nội dung sau:

a) Tiền xư lý ảnh Sentinel-1A

Ảnh vệ tinh Sentinel-1A được chuyển đổi giá trị số sang giá trị tán xạ ngược của ảnh rađa [24]. Bộ lọc Lee [25] một trong những phương pháp lọc không gian được áp dụng trong nghiên cứu này nhằm giảm hiện tượng muối tiêu và những tín hiệu không đồng nhất tại bề mặt của nước biển. Tiếp theo, mô hình số độ cao toàn cầu SRTM được sử dụng nhằm mục đích hiệu chỉnh các biến dạng hình học. Hệ tọa độ World Geodetic System 84 (WGS 84) và phép chiếu UTM zone 49 North được thiết lập cho dữ liệu ảnh vệ tinh Sentinel-1A (Hình 2).
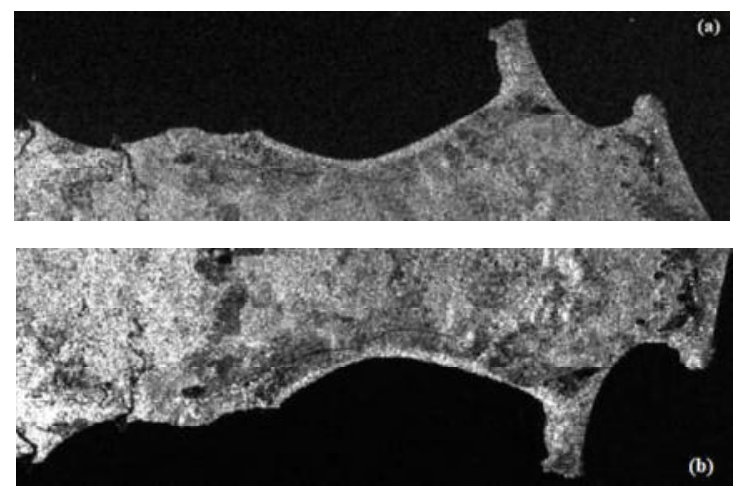

Hình 2. Anh vệ tinh Sentinel-1A trước (a) và sau (b) khi tiền xủ lý 


\section{b) Xác định ranh giới giữa đất và nước}

Ranh giới giữa đất và nước được xác định thông qua quy trình gồm hai bước: phân cụm mờ và thiết lập ngưỡng. Đầu tiên, các hàm phân cụm mờ được sử dụng để đưa các giá trị pixel về ngưỡng từ 0 đến 1 . Do giá trị trung bình và độ lệch chuẩn giữa các pixel đất và nước là rất lớn, hàm phân cụm mờ được thiết lập theo công thức (1) nhằm làm tối ưu hóa độ phân tán dữ liệu:

$u(x)=1-\frac{b s}{x-a m+b s}$ if $x>$ am otherwise $\mu(x)=0$

Trong đó: $\mathrm{m}$ = giá trị trung bình; $\mathrm{s}$ = độ lệch chuẩn; $\mathrm{b}$ và a là các thông số thực nghiệm.

Giá trị trung bình và độ lệch chuẩn trên ảnh Sentinel 1A tại khu vực nghiên cứu với kết quả lần lượt là: $\mathrm{m}=0,31$ và $\mathrm{s}=0,44$.

Để khởi tạo hàm phân cụm mờ, một loạt các giá trị thực nghiệm được sử dụng để lựa chọn thông số $a$ và $b$ phù hợp với khu vực nghiên cứu. Kết quả thực nghiệm cho thấy $\mathrm{a}=0,43$ và $\mathrm{b}=$ 0,04 là phù hợp để phân biệt tốt đối tượng đất và nước tại khu vực nghiên cứu. Kết quả áp dụng hàm phân cựm mờ cho thấy vùng có giá trị pixel càng gần đến 1 thì có màu càng sáng, vùng có giá trị pixel càng gần đến 0 thì có màu càng tối (Hình 3).

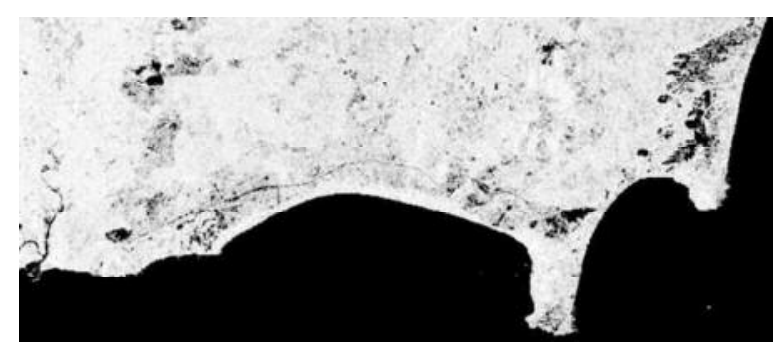

Hình 3. Kết quả áp dụng hàm phân cụm mò̀.

Sau khi áp dụng hàm phân cụm mờ thì ranh giới giữa đất và nước trên ảnh được phân biệt rõ ràng. Phương pháp được sử dụng tiếp theo trong quy trình trích xuất đường bờ là việc áp dụng ngưỡng thích hợp để phân đoạn ảnh. Dựa trên biểu đồ hiển thị dạng phân phối tần suất các giá trị pixel của kết quả phân cụm mờ, giá trị ngưỡng tối ưu 0,502 được sử dụng để tạo ảnh nhị phân gồm 2 đối tượng đất và nước. Các giá trị pixel trên ngưỡng 0,502 sẽ được phân vào lớp đất, ngược lại là lớp nước. Phương pháp này tạo ra sản phẩm là một ảnh nhị phân có chứa các giá trị pixel là 1 (lớp đất) hoặc 0 (lớp nước).

Ranh giới giữa đất và nước trên ảnh nhị phân được xác định để trích xuất đường bờ (Hình 4).

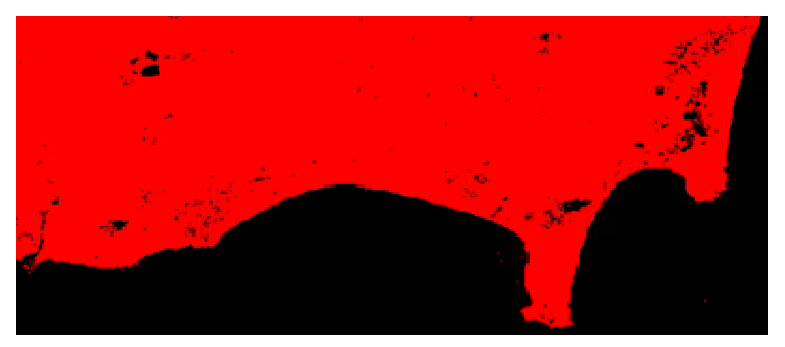

Hình 4. Kết quả áp dụng nguỡng để phân tách giữa đất (màu đỏ) và nước (màu đen)

c) Trích xuất dũ liệu đường bò̀ biển

Từ kết quả xác định ranh giới giữa đất và nước, dữ liệu được chuyển sang dạng véc tơ. Kỹ thuật khái quát hóa đối tượng trong GIS được sử dụng để loại bỏ hiệu ứng zic zắc của đường bờ (Hình 5).

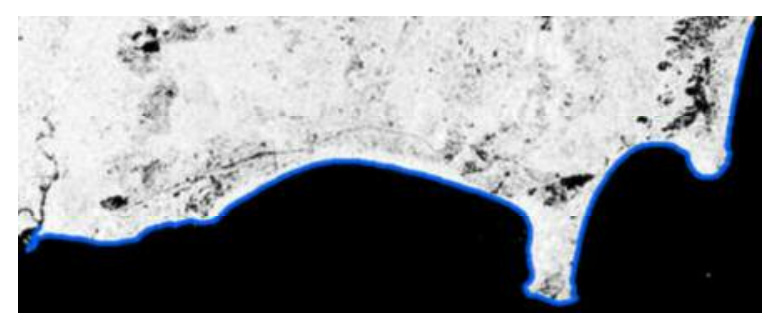

Hình 5. Kết quả đường bò̀ biển được trich xuất tù ảnh vệ tinh Sentinel-1A

d) Đánh giá độ chính xác của đường bò̀ biển được trích xuất

Kết quả đường bờ trích xuất từ phương pháp phân cụm mờ - thiết lập ngưỡng được đánh giá độ chính xác với đường bờ được số hóa thủ công, bằng cách tính toán khoảng cách vuông góc giữa 2 đường.

Nhằm trích xuất thông tin đối tượng đường bờ biển từ phương pháp số hóa thủ công (Digitizing), dữ liệu ảnh vệ tinh độ phân giải cao Google Earth với độ phân giải không gian $1 \mathrm{~m}$ được hiển thị trên phần mềm GIS thông qua công cụ HCMGIS. Ảnh Google Earth được số hóa thủ công dựa trên việc nhận biết sự khác biệt về tính chất phản xạ của đối tượng đất và nước. Kết quả đường bờ biển được trích xuất từ 
phương pháp số hóa được thể hiện như trong Hình 6.

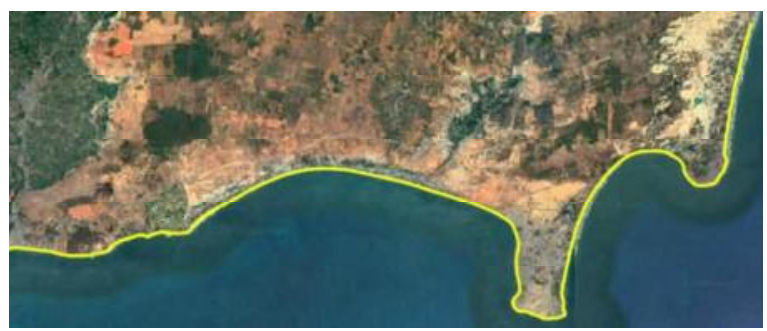

Hình 6. Kết quả đường bò̀ biển được trích xuất tù ảnh Google Earth bằng phương pháp số hóa

Công cụ DSAS (Digital Shoreline Analysis System) được sử dụng để tính toán sự khác biệt giữa tỷ lệ đường bờ trích xuất từ phương pháp phân cựm mờ - thiết lập ngưỡng và đường bờ được số hóa thủ công. 350 đoạn thẳng cách đều nhau $100 \mathrm{~m}$ dùng để tính khoảng cách giữa 2 đường bờ (Hình 7).

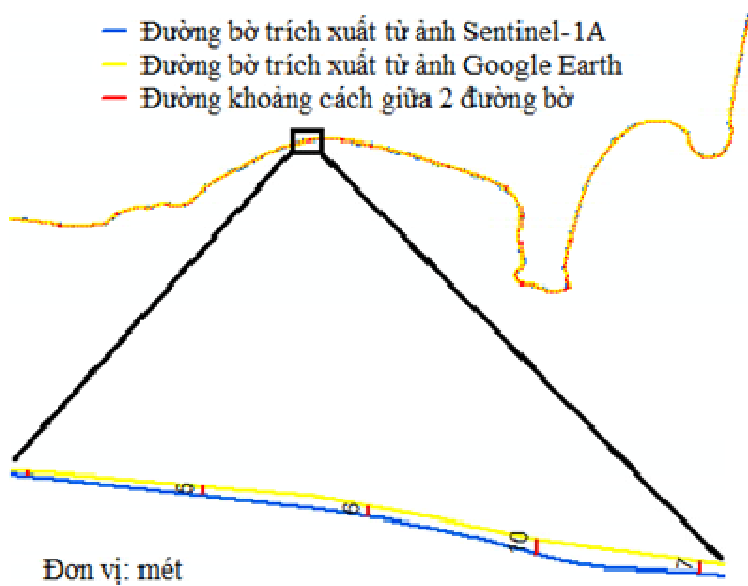

Hình 7. Vị trí các đường khoảng cách giũa 2 đường bò được xác định bằng công cu DSAS

\section{Kết quả và thảo luận}

Thống kê cho 350 vị trí tính khoảng cách giữa 2 kết quả đường bờ được thể hiện như trong Hình 8.

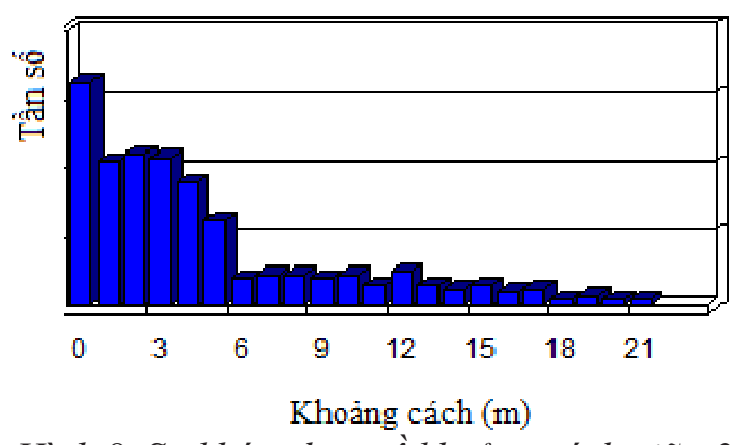

Hình 8. Sụ khác nhau về khoảng cách giữa 2 đưòng bò

Trong đó, 298 (85\%) vị trí có độ sai khác giữa 2 đường bờ từ $0-10 \mathrm{~m}$ (tương đương với sai số 1 pixel trên ảnh Sentinel-1A), 52 (15\%) vị trí có độ sai khác giữa 2 đường bờ từ $11-22 \mathrm{~m}$. Khoảng cách sai khác trung bình và cao nhất giữa 2 kết quả trích xuất đường bờ lần lượt là 4,7 $\mathrm{m}$ và $22 \mathrm{~m}$. Từ đó cho thấy kết quả trích xuất đường bờ bằng phương pháp phân cụm mờ và thiết lập ngưỡng từ ảnh vệ tinh Sentinel-1A đạt độ chính xác cao, có khả năng phân biệt rõ ranh giới giữa đối tượng đất và nước.

\section{Kết luận}

Kết quả trích xuất đường bờ bằng phương pháp phân cụm mờ áp dụng cho ảnh vệ tinh Sentinel-1A tại khu vực ven biển TP. Phan Thiết cho kết quả tương đồng với phương pháp giải đoán bằng mắt thường. Phương pháp này có ưu điểm nổi bật là đường bờ được trích xuất một cách tự động. Thách thức của phương pháp này là việc chọn các thông số thực nghiệm và xác lập ngưỡng tối ưu để trích xuất thông tin đường bờ. Chúng tôi tin rằng, với nguồn ảnh ra đa Sentinel1 A miễn phí và các phương pháp rút trích đường bờ cải tiến sẽ giúp cho các nhà khoa học có thể đánh giá nhanh sự biến động đường bờ một cách liên tục trong phạm vi lớn. Từ đó, đưa ra được các phương án hợp lý với tình trạng xói lở và bồi tụ ở khu vực ven biển.

\section{Tài liệu tham khảo}

1. Nguyễn Đình Vượng (2017). Đánh giá quá trình xâm thực bờ biển tỉnh Bình Thuận - Phân tích nguyên nhân và đề xuất giải pháp phòng chống, Tạp chí Khoa học kỹ thuật Thủy lợi và Môi trường, no. 12 , p. 84.

2. Phạm Bá Trung (2011). Hiện trạng Xói lở - Bồi tụ bờ biển tỉnh Bình Thuận, Vietnam journal of earth sciences, vol. 33, no. 3, pp. 322 - 328. 


\section{BÀI BÁO KHOA HỌC}

3. Huỳnh Trung Tín, Yoshiaki Nishikawa, Nguyễn Thành Luân, and Bùi Trọng Vinh, Co chếxói lở bãi biển Đồi Dương, Tp. Phan Thiết và đề xuất giải pháp phòng chống.

4. Robert, D., Hayden, B.P., May, P. and May,S. (1980). The reliability of shoreline change measurements from aerial photographs, Shore and beach, vol. 48, no. 4, pp. 22 - 29.

5. Elizabeth, H.B. and Turner, I.L. (2005). Shoreline definition and detection: a review, Journal of coastal research, pp. $688-703$.

6. Stephen, P. (1983). Shoreline mapping: a comparison of techniques, Shore and Beach, vol. 51, no. 3 , pp. 28 - 33.

7. Ron, L., Di.K. and Ma,R. (2001). A comparative study of shoreline mapping techniques, GIS for coastal zone management, pp. 53 - 60.

8. Robert, D., Fenster,M.S., and Stuart Holme,J. (1991). Temporal analysis of shoreline recession and accretion, Journal of coastal research, pp. 723 - 744.

9. Offer,R., Siegal,Z.Blumberg,D.G., and Adamowski,J. (2016) Investigating the backscatter contrast anomaly in synthetic aperture radar (SAR) imagery of the dunes along the Israel-Egypt border, International journal of applied earth observation and geoinformation, vol. 46, pp. 13 - 21 .

10. Harold,C.M., Lewis,J.A., and Wing,R. (1971). Mapping and LandformAnalysis of Coastal Regions with Radar, Geological Society of America Bulletin, vol. 82, no. 2, pp. 345 - 358.

11. H,L., and Jezek,K. (2004). Automated extraction of coastline from satellite imagery by integrating Canny edge detection and locally adaptive thresholding methods, International Journal of remote sensing, vol. 25, no. 5, pp. 937 - 958.

12. Andrea,B., Nunziata,F., Mascolo,L., and Migliaccio,M. (2014). A multipolarization analysis of coastline extraction using X-band COSMO-SkyMed SAR data, IEEE Journal of Selected Topics in Applied Earth Observations and Remote Sensing, vol. 7, no. 7, pp. 2811 - 2820.

13. Sheng,G., Yang,W., Deng,X., He,C., Cao,Y., and Sun,H. (2012) Coastline detection in synthetic aperture radar (SAR) images by integrating watershed transformation and controllable gradient vector flow (GVF) snake model, IEEE Journal of Oceanic Engineering, vol. 37, no. 3, pp. 375 $-383$.

14. Ireena,A,E. (1998). An automatic coastline detector for use with SAR images, Sandia National Laboratories (SNL - NM), Albuquerque, NM.

15. Łukasz,M., Mazurek,P., and Chybicki,A. (2016). Coastline change - detection method using remote sensing satellite observation data, Hydroacoustics, vol. 19.

16. Filsa,B., and Hayati,N. (2016). Coastline changes detection using Sentinel-1 satellite imagery in Surabaya, East Java, Indonesia, Geoid, vol. 11, no. 2, pp. 190 - 198.

17. Hongwei,Zhang., Zhang,B., Guo,H., Lu,J., and He,H. (2013). An automatic coastline extraction method based on active contour model, in Geoinformatics (GEOINFORMATICS), 2013 21st International Conference on, pp. 1 - 5: IEEE.

18. Rafael, LP., Nunziata,F., and Migliaccio,M. (2015). Coastline extraction and coastal area classification via SAR hybrid-polarimetry architecture, in Geoscience and Remote Sensing Symposium (IGARSS), 2015 IEEE International, pp. 3798 - 3801: IEEE.

19. Mohammad,M., and Akbarizadeh,G. (2017). Coastline extraction from SAR images using spatial fuzzy clustering and the active contour method, International journal of remote sens ing, vol. 38 , no. 2, pp. 355 - 370.

20. Zhongling, L., Li,F., Li,N., Wang,R., and Zhang,H. (2016). A novel region-merging approach for coastline extraction from Sentinel-1A IW mode SAR imagery, IEEE Geoscience and remote sensing letters, vol. 13, no. 3, pp. 324 - 328. 
21. N,Demir., Kaynarcaa,M., and Oya,S. (2016). Extraction of coastlines with fuzzy approach using SENTINEL-1 SAR image, ISPRS - International Archives of the Photogrammetry, Remote Sensing and Spatial Information Sciences, vol. 1, pp. 747 - 751.

22. N,D., Oy,S., Erdem,F., Şeker,DZ., and Bayram,B. (2017). Integrated shoreline extraction approach with use of Rasat MS and Sentinel-1a SAR images, ISPRS Annals of Photogrammetry, Remote Sensing \& Spatial Information Sciences, vol. 4.

23. Hồng Long Bùi (2004). Một số kết quả khảo sát, nghiên cưu hiện tượng xói lỏ, bồi tụ khu vưc ven biển Bình Thuận Some studied results on erosion and deposition in the coastal area of Binh Thuan province.

24. N,M., Meadows,PJ., TYPE,D., and NOTE,T. (2015). Radiometric Calibration of S-1 Level1 Products Generated by the S-l IPF, Viewed at https://sentinel.esa.int/documents/247904/685163/S1- Radiometric-Calibration-V1. 0. pdf.

25. Jong,SL. (1986). Speckle suppression and analysis for synthetic aperture radar images, Optical engineering, vol. 25, no. 5, p. 255636.

\title{
THE EXTRACTION OF SHORELINE USING THE SENTINEL-1A SATELLITE IMAGERY IN PHAN THIET CITY Huynh Yen Nhi ${ }^{1}$, Le Thi Kim Thoa ${ }^{2}$ \\ ${ }^{1,2}$ University of Natural Resources and Environment
}

\begin{abstract}
In recent years, erosions (or accretion processes) have been occurring in coastal areas. Remote sensing is used to measure the shoreline morphology for a long time. In this study, Sentinel1 A satellite imagery is used to extract the shoreline in Phan Thiet City. The boundary between land and water is determined by a two-step process: fuzzy clustering and interactive thresholding. The results of shoreline extraction are extracted into vector form. This shoreline is compared to manually digitized shoreline. There are 350 locations considered to determine the distance between two shorelines, of which 298 locations (85\%) are 0 to $10 \mathrm{~m}$ (equivalent to 1 pixel of Sentinel-1A) and 52 (85\%) locations are over $10 \mathrm{~m}$. The study results show that the ability to apply fuzzy clustering and set thresholds to extract the shoreline automatically on Sentinel-1A images is effective for rapid assessment of coastal erosion and accretion in coastal area.
\end{abstract}

Keywords: Erosion/Accretion, Shoreline extraction, Sentinel-1A, Fuzzy clustering and Interactive thresholding. 